\title{
High expression of 5-hydroxymethylcytosine and isocitrate dehydrogenase 2 is associated with favorable prognosis after curative resection of hepatocellular carcinoma
}

\author{
Wei-Ren Liu, ${ }^{1,2 \dagger}$, Meng-Xin Tian 1,2†, Lei Jin ${ }^{1,2}$, Liu-Xiao Yang ${ }^{1,2}$, Zhen-Bin Ding ${ }^{1,2}$, Ying-Hao Shen ${ }^{1,2}$, Yuan-Fei Peng ${ }^{1,2}$,
} Jian Zhou ${ }^{1,2,3}$, Shuang-Jian Qiu, ${ }^{1,2}$, Zhi Dai ${ }^{1,2}$, Jia Fan ${ }^{1,2,3^{*}}$ and Ying-Hong Shi ${ }^{1,2^{*}}$

\begin{abstract}
Background: The expression of 5-hydroxymethylcytosine (5-hmC) and isocitrate dehydrogenase 2 (IDH2) is frequently downregulated in numerous cancers. 5-hmC and IDH2 expression in hepatocellular carcinoma (HCC) has yet to be determined.

Methods: The immunohistochemical expression of 5-hmC and IDH2 were analyzed in tissue microarrays containing samples from 646 patients who had undergone hepatectomy for histologically proven HCC. The prognostic value of 5-hmC and IDH2 were evaluated by Cox regression and Kaplan-Meier analyses.

Results: We discovered that low 5-hmC and IDH2 expression was associated with malignant behaviors. Low 5-hmC or IDH2 expression alone and combined 5-hmC and IDH2 expression were associated with lower overall survival (OS) rates and higher cumulative recurrence rates. Multivariate analysis indicated that 5-hmC or IDH2 and 5-hmC/IDH2 were independent prognostic indicators for OS and time to recurrence (TTR), which was confirmed in an independent validation cohort.
\end{abstract}

Conclusions: $5-\mathrm{hmC}$ and IDH2 correlate with less aggressive tumor behavior in HCC. When 5-hmC and IDH2 are considered together, they serve as a prognostic marker in patients with surgically resected HCCs.

Keywords: 5-hydroxymethylcytosine, Isocitrate dehydrogenase 2, Hepatocellular carcinoma, Immunohistochemistry, Prognosis

\section{Background}

Liver cancer is the fifth most common cancer in men and the seventh most common in women, and hepatocellular carcinoma (HCC) is diagnosed in more than half a million of these liver cancer patients worldwide each year [1]. Despite intensive research, the prognosis of HCC remains poor, with an overall 5-year survival rate of approximately $26 \%$ in the United States [2]. There is a pressing need for novel biomarkers to identify the subset

\footnotetext{
*Correspondence: fan.jia@zs-hospital.sh.cn; shi.yinghong@zs-hospital.sh.cn ${ }^{\dagger}$ Equal contributors

'Department of Liver Surgery, Liver Cancer Institute, Zhongshan Hospital, Fudan University, 180 FengLin Road, Shanghai 200032, China

${ }^{2}$ Key Laboratory of Carcinogenesis and Cancer Invasion of Ministry of Education, Zhongshan Hospital, Fudan University, 180 FengLin Road, Shanghai, China Full list of author information is available at the end of the article
}

of patients with a high risk of recurrence and/or poor survival outcomes.

In the current cancer research landscape, epigenetics is a promising and expanding field [3-6]. DNA methylation, an important pattern of epigenetics, was historically believed to be a relatively stable chromatin modification, but the detection of the presence of 5 -hmC facilitated a breakthrough in the field of epigenetic research [7,8]. 5$\mathrm{hmC}$, also known as the "sixth base", was identified as an oxidant product of 5-methylcytosine $(5 \mathrm{mC})$ via the ten-eleven translocation (TET) family, which consists of TET1, -2 , and -3 . 5 -hmC is abundant in embryonic stem (ES) cells and adult neural cells [8-10]. Currently, the biological prevalence of $5-\mathrm{hmC}$ in cancer remains elusive. Lian et al. reported that the loss of $5-\mathrm{hmC}$ was 
an epigenetic characteristic of melanoma with diagnostic and prognostic efficiency [11]. 5-hmC levels were high in low-grade tumors and decreased in malignant glioma [12]. Regarding gastroenteric tumors, 5-hmC was decreased in colorectal cancer (CRC) and gastric cancer [13]. In liver cancer, 5-hmC was also decreased compared with the surrounding normal tissue [14-16].

Isocitrate dehydrogenases (IDHs) catalyze the oxidative decarboxylation of isocitrate, which converts isocitrate to $\alpha$-ketoglutarate (KG). The IDHs include IDH1 in the cytoplasm and IDH2 in the mitochondria, which catalyze an identical reaction [17] (Additional file 1: Figure S1). IDH1 and IDH2 mutations widely occur in gliomas and acute myeloid leukemia [18-21], leading to the production of 2hydroxyglutarate (2-HG), which inhibits multiple $\alpha-K G$ dependent dioxygenases, including the TET family of 5 -mC hydroxylases (which results in decreased 5-hmC) [22]. Lian et al. found that IDH2 was significantly downregulated in melanoma [11]. However, 5-hmC and IDH2 expression in HCC have yet to be characterized in a large series of tumors with documented clinical, pathological, and molecular information.

In this study, we sought to determine the clinical relevance of 5 -hmC and IDH2 protein expression in a large series of surgically resected HCCs using two cohorts. We studied the association between these two proteins and tumor history, as well as the patients' clinical-pathologic features, including age, sex, stage, overall survival (OS), and time to recurrence (TTR). We found that combined 5-hmC and IDH2 protein expression was an independent prognostic factor for $\mathrm{HCC}$ patients after surgery.

\section{Materials and methods}

\section{Patients and specimens}

Archival specimens were obtained from two cohorts of consecutive patients with HCC who underwent curative resection at the Liver Cancer Institute, Zhongshan Hospital, Fudan University, between 2006 and 2007. The patient cohort inclusion and exclusion criteria included (a) accurate pathologic diagnosis of HCC, (b) complete clinicopathologic and follow-up data, (c) no anticancer treatment prior to curative liver resection, and (d) complete formalin-fixed, paraffin-embedded tissues. The histopathological diagnosis was determined according to the World Health Organization criteria. Tumor differentiation was graded using the Edmondson grading system [23]. Tumor staging was based on the 6th edition of the tumor-node-metastasis (TNM) classification of the International Union Against Cancer. Most patients (82.4\%) had a hepatitis B virus background, and only two patients had hepatitis $C$ virus. Almost all patients (316 of 318 for the training cohort and 325 of 328 for the validation cohort) were in the Child-Pugh A classification. The clinicopathologic characteristics of the two cohorts are summarized in
Additional file 2: Table S1. Ethical approval was obtained from the Zhongshan Hospital Research Ethics Committee, and written informed consent was obtained from each patient.

\section{Follow-up and postoperative treatment}

The follow-up data were summarized at the end of December 2011, with a median observation time of 52.2 months. The follow-up procedures were described in our previous study [23,24]. Postsurgical patient surveillance was undertaken as previously described [23,25]. OS was defined as the interval between the dates of surgery and death. TTR was defined as the interval between the dates of surgery and the dates of any diagnosed recurrence (intrahepatic recurrence and extrahepatic metastasis). For surviving patients, the data were censored at the date of death or last follow-up.

\section{Tissue microarray and immunohistochemistry}

Tissue microarray (TMA) was conducted as previously described [26-28]. Briefly, all samples from the HCC patients were reviewed by three histopathologists and representative areas located away from necrotic and hemorrhagic materials were premarked in the paraffin blocks. Two core biopsies (1 $\mathrm{mm}$ in diameter) were taken from each representative tumor tissue and peritumoral tissue to construct the TMA slides. Consecutive sections measuring $4 \mu \mathrm{m}$ were placed on 3-aminopropyltriethoxysilane-coated slides (Shanghai Biochip Co Ltd, Shanghai, People's Republic of China).

Immunohistochemistry of the paraffin sections was performed using a two-step protocol (Novolink Polymer Detection System, Novocastra) according to the manufacturer's instructions. Briefly, paraffin-embedded sections were deparaffinized and then rehydrated; after heat-induced antigen retrieval, endogenous peroxidases were blocked for 5 min using $0.3 \% \mathrm{H}_{2} \mathrm{O}_{2}$, washed twice, and then incubated for $5 \mathrm{~min}$ in Serum-Free Protein Block (Novocastra), followed by incubation for $60 \mathrm{~min}$ in purified rabbit antihuman 5-hmC and rabbit anti-human IDH2 with DaVinci Green antibody diluent (Biocare Medical). The sections were incubated in a 3, 3-diaminobenzidine solution, counterstained with hematoxylin, dehydrated in ethanol, cleared in xylene, and coverslipped. Negative controls were treated in all assays (with the omission of primary antibodies). The sections were visualized using microscopic observation.

\section{Evaluation of the immunohistochemical findings}

IHC staining was assessed by two independent pathologists without knowledge of the clinical and pathologic features of the cases. A negative control array was concurrently undertaken showing $<1 \%$ nuclear staining in all specimens. All specimens were evaluated according to the $0-4$ grading criteria (based on the percentage of 5 -hmC-positive cells) and $0-3$ grading criteria (based on the staining intensity) 
[11]. The $5-\mathrm{hmC}$ score was calculated as the score of the cell count $x$ the score of intensity. The median 5 -hmC score was used as a cut-off in subsequent analyses. For IDH2 quantification, photographs of three representative fields were captured under high-power magnification (200x) using Leica Qwin Plus v3 software; identical settings were used for each photograph. The 5-hmC and IDH2 density were counted using Image-Pro Plus v6.2 software (Media Cybernetics Inc., Bethesda, MD). The integrated optical density of the area positively stained for IDH2 in each photograph was calculated, and its ratio to the total area of each photograph was considered to be the IDH2 density. The median IDH2 density was used as a cut-off in subsequent analyses.

\section{Statistical analysis}

The data were analyzed with SPSS 19.0 software, as previously described [23]. A $P$ value $<0.05$ was considered statistically significant.

\section{Results}

Immunohistochemical features in TMA

Using hematoxylin and eosin staining, the cancer cells were found to be relatively homogenous within a tumor (excluding necrotic, hemorrhagic, and fibrotic components). Representative cases of immunohistochemical staining are shown in Figure 1. We observed 5-hmC staining primarily on the nuclei of the tumor cells and hepatocytes; IDH2 staining was observed primarily in the cytoplasm of the HCC cells. Most of the stromal cells were negatively stained, although sporadic positive staining of these cells was observed.

\section{Correlations of 5-hmC and IDH2 expression with clinico- pathologic characteristics}

The correlations of 5-hmC and IDH2 expression with the clinicopathologic characteristics are shown in Table 1 and Additional file 2: Table S2. In the training cohort, 5hmC expression correlated with sex $(P=0.007)$ and AFP
$(P<0.001)$. IDH2 expression only correlated with tumor differentiation $(P=0.017)$ (Table 1$)$. In the validation cohort, 5-hmC expression correlated with sex $(P=0.003)$, age $(P=0.034)$, AFP $(P<0.001)$, tumor number $(P=0.02)$, and TNM stage $(P=0.009)$. IDH2 expression correlated with HBsAg $(P=0.015)$, AFP $(P<0.001)$, and tumor differentiation $(P=0.015)$ (Additional file 2: Table S2). Other clinical characteristics were not directly related to the expression of 5-hmC or IDH2.

\section{Association between combined 5-hmC and IDH2 expression and outcome in the training cohort}

By the last follow-up in the training cohort (November 2011), $47.2 \%$ (150/318) of the patients had suffered a recurrence and 36.5\% (116/318) had died. The 1-, 3-, and 5 -year OS rates in the cohort were $83.6 \%, 67.6 \%$, and $63.5 \%$ and the cumulative recurrence rates were $32.7 \%$, $46.9 \%$, and $52.8 \%$, respectively. Additionally, we found that the 1-, 3-, and 5-year survival rates of the 5-hmC High patients were significantly higher than those of the 5 -hmC ${ }^{\text {Low }}$ group $(87.4 \%$ vs. $79.9 \%, 77.4 \%$ vs. $57.9 \%$, and $73.0 \%$ vs. $54.1 \%$, respectively) (Figure $2 \mathrm{a}$ ). Similarly, the 5 -hmC ${ }^{\text {Low }}$ patients had a poorer prognosis at 1,3 , and 5 years, with higher cumulative recurrence rates than the 5 -hmC ${ }^{\text {High }}$ patients $(40.3 \%$ vs. $25.2 \%, 56.6 \%$ vs. $37.1 \%$, and $61.6 \%$ vs. $44.0 \%$, respectively) (Figure 2b). We also discovered that the 1-, 3-, and 5-year survival rates of the IDH2 ${ }^{\text {High }}$ patients were significantly higher than those of the IDH2 ${ }^{\text {Low }}$ group ( $93.7 \%$ vs. $73.6 \%, 76.7 \%$ vs. $58.5 \%$, and $71.7 \%$ vs. $55.3 \%$, respectively) (Figure 2a). Similarly, the IDH2 ${ }^{\text {Low }}$ patients had a poorer prognosis at 1-, 3-, and 5 - years, with higher cumulative recurrence rates than the IDH2 ${ }^{\text {High }}$ patients $(40.3 \%$ vs. $25.2 \%, 52.2 \%$ vs. $41.5 \%$, and $58.5 \%$ vs. $47.2 \%$, respectively) (Figure 2b). Furthermore, we evaluated the combined effect of 5 -hmC and IDH2 expression. We found that the 1-, 3-, and 5-year OS rates in the 5 -hmC ${ }^{\text {Low } / I D H 2 ~}{ }^{\text {Low }}$ patients were $64.6 \%, 43.1 \%$, and $43.1 \%$, respectively, which were significantly lower than those in the 5-hmC ${ }^{\mathrm{High}} / \mathrm{IDH} 2{ }^{\mathrm{High}}$ patients $(98.5 \%, 89.2 \%$,

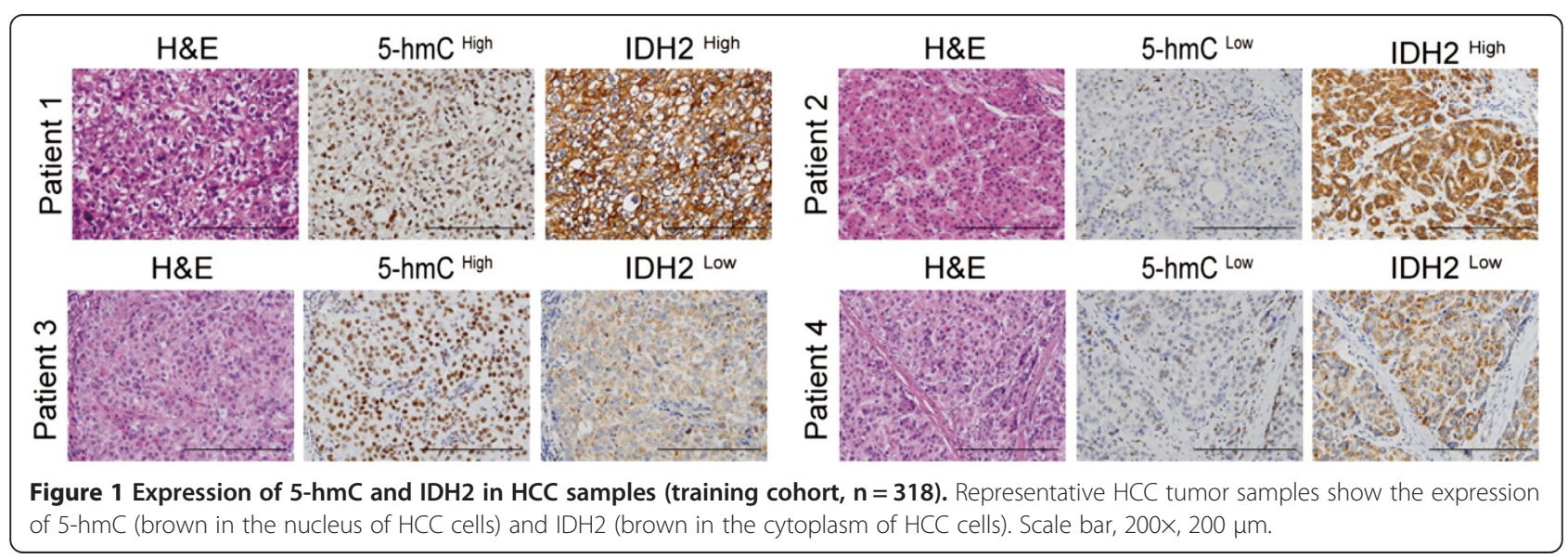


Table 1 Summary of the correlations of 5-hmC and IDH2 protein expression with clinicopathological features in the training cohort $(\mathrm{N}=\mathbf{3 1 8})$

\begin{tabular}{|c|c|c|c|c|c|c|c|}
\hline \multirow[t]{2}{*}{ Clinicopathological indexes } & & \multicolumn{3}{|c|}{ No. of patients } & \multicolumn{3}{|c|}{ No. of patients } \\
\hline & & 5-hmC Low & 5-hmC High & $\mathrm{P}^{+}$ & IDH2 Low & IDH2 ${ }^{\text {High }}$ & $\mathrm{P}^{+}$ \\
\hline \multirow[t]{2}{*}{ Sex } & Female & 18 & 36 & 0.007 & 28 & 26 & 0.765 \\
\hline & Male & 141 & 123 & & 131 & 133 & \\
\hline \multirow[t]{2}{*}{ Age(year) } & $\leq 50$ & 55 & 65 & 0.247 & 60 & 60 & 1.000 \\
\hline & $>50$ & 104 & 94 & & 99 & 99 & \\
\hline \multirow[t]{2}{*}{$\mathrm{HBsAg}$} & Negative & 30 & 26 & 0.556 & 28 & 28 & 1.000 \\
\hline & Positive & 129 & 133 & & 131 & 131 & \\
\hline \multirow[t]{2}{*}{$\mathrm{HCV}$} & Negative & 158 & 158 & 1.000 & 157 & 159 & 0.156 \\
\hline & Positive & 1 & 1 & & 2 & 0 & \\
\hline \multirow[t]{2}{*}{ AFP } & $\leq 20$ & 83 & 37 & $<0.001$ & 58 & 62 & 0.644 \\
\hline & $>20$ & 76 & 122 & & 101 & 97 & \\
\hline \multirow[t]{2}{*}{$\gamma-G T(U / L)$} & $\leq 54$ & 87 & 81 & 0.500 & 78 & 90 & 0.178 \\
\hline & $>54$ & 72 & 78 & & 81 & 69 & \\
\hline \multirow[t]{2}{*}{ Liver cirrhosis } & No & 32 & 26 & 0.384 & 23 & 35 & 0.081 \\
\hline & Yes & 127 & 133 & & 136 & 124 & \\
\hline \multirow[t]{2}{*}{ Tumor number } & Single & 131 & 134 & 0.652 & 134 & 131 & 0.652 \\
\hline & Multiple & 28 & 25 & & 25 & 28 & \\
\hline \multirow[t]{2}{*}{ Tumor size(cm) } & $\leq 5$ & 97 & 108 & 0.197 & 99 & 106 & 0.412 \\
\hline & $>5$ & 62 & 51 & & 60 & 53 & \\
\hline \multirow[t]{2}{*}{ Tumor encapsulation } & Complete & 94 & 88 & 0.496 & 93 & 89 & 0.650 \\
\hline & None & 65 & 71 & & 66 & 70 & \\
\hline \multirow[t]{2}{*}{ Microvascular invasion } & Absent & 113 & 107 & 0.466 & 106 & 114 & 0.331 \\
\hline & Present & 46 & 52 & & 53 & 45 & \\
\hline \multirow[t]{2}{*}{ Tumor differentiation } & $I+\|$ & 129 & 115 & 0.063 & 113 & 131 & 0.017 \\
\hline & $I I I+I V$ & 30 & 44 & & 46 & 28 & \\
\hline \multirow[t]{2}{*}{ TNM stage } & 1 & 98 & 93 & 0.567 & 93 & 98 & 0.567 \\
\hline & $\|+\| I I$ & 61 & 66 & & 66 & 61 & \\
\hline
\end{tabular}

Abbreviations: HBsAg, hepatitis B surface antigen; AFP, a-fetoprotein; $\gamma$-GT, $\gamma$-glutamyl transferase; TNM, tumor-node-metastasis.

${ }^{\dagger} A P$-value $<0.05$ was considered statistically significant. $P$-values were calculated using the Pearson chi-square test. Boldface type indicates significant values.

and $86.2 \%$, respectively) (Figure $2 \mathrm{a}$ ). The cumulative recurrence rates in the 5 -hmC ${ }^{\text {Low }} / \mathrm{IDH} 2{ }^{\text {Low }}$ patients were $52.3 \%, 63.1 \%$ and $66.2 \%$, respectively, which were significantly higher than those in the 5 -hmC $\mathrm{High} / \mathrm{IDH} 2$ High patients (15.4\%, 26.2\% and 30.8\%, respectively) (Figure $2 \mathrm{~b}$ ).

Univariate analysis revealed that $5-\mathrm{hmC}(P<0.001$ and $P=0.001)$, IDH2 $(P<0.001$ and $P=0.006)$, and $5-\mathrm{hmC} /$ IDH2 combined $(P<0.001$ and $P<0.001)$ were associated with OS and TTR. $\gamma$-GT, tumor number, tumor size, microvascular invasion, and TNM stage were predictors of OS and TTR. Moreover, AFP was only associated with OS, and liver cirrhosis was only associated with TTR (Table 2).

The individual clinicopathological features that presented significance in the univariate analysis were adopted as covariates in a multivariate Cox proportional hazards model for further analysis. 5-hmC and IDH2 were prognostic indicators of OS $(P<0.001$ and $P<0.001)$ and TTR ( $P<0.001$ and $P=0.001)$. When 5 -hmC was combined with IDH2, we found that 5 -hmC/IDH2 was also an independent prognostic indicator of both OS $(P<0.001)$ and $\operatorname{TTR}(P<0.001)$ (Figure 2 and Table 2$)$.

Validation analysis of the better outcome of patients in the validation cohort with $5-\mathrm{hmC}^{\mathrm{High}} / \mathrm{IDH} 2{ }^{\mathrm{High}}$ expression

To validate our findings of better outcomes in patients with 5-hmC High/IDH2 High expression, we studied a validation cohort that included 328 surgically resected HCC tumors. Briefly, we found that the 1- and 3-year OS rates in the $5-\mathrm{hmC}{ }^{\text {Low }} / \mathrm{IDH} 2{ }^{\text {Low }}$ patients were $66.3 \%$ and $46.3 \%$, respectively, which were significantly lower than those in the 5-hmC High/IDH2 High patients (97.0\% and $79.0 \%$, respectively) (Figure 3a). The cumulative 

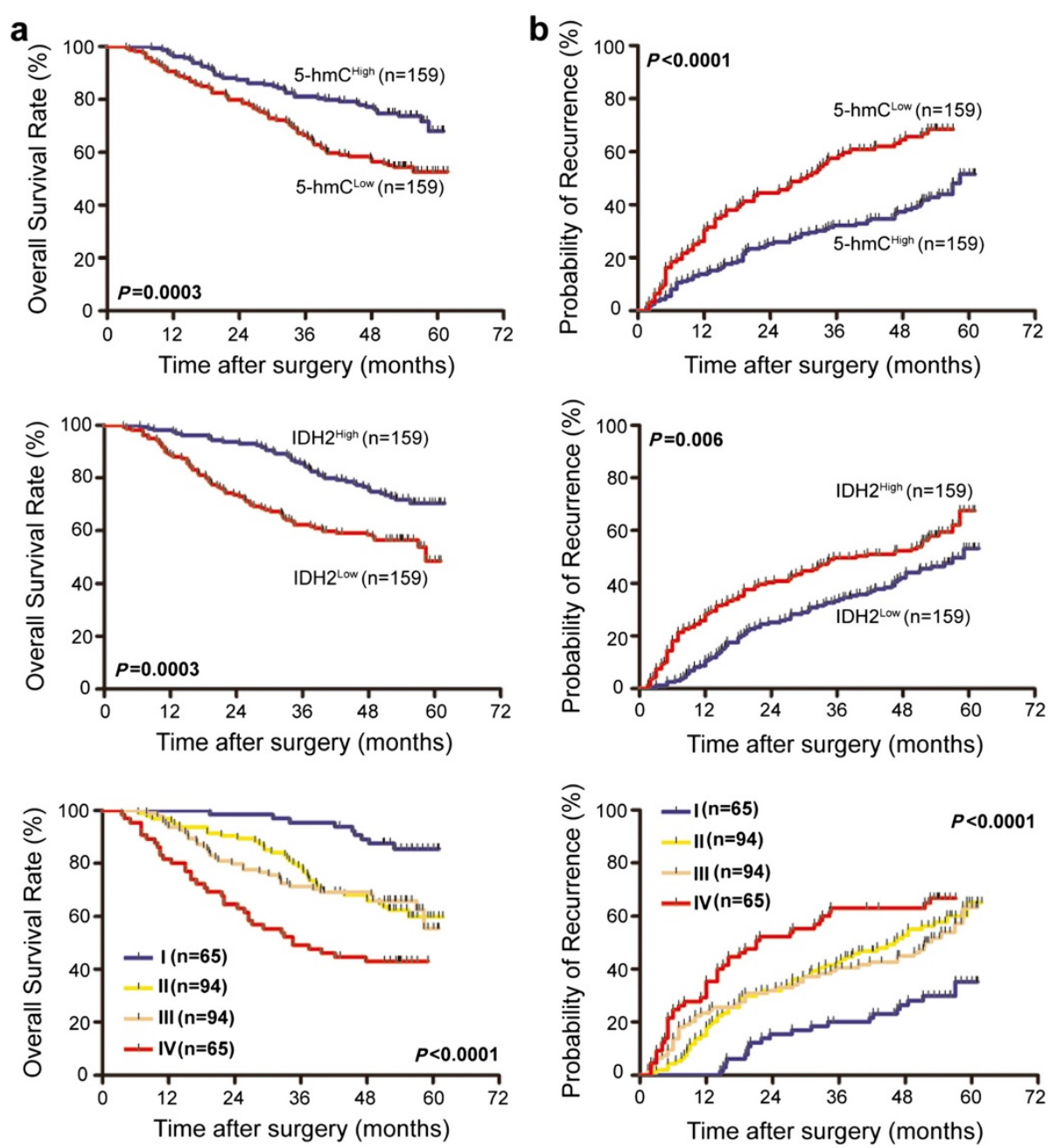

Figure 25 -hmC and IDH2 expression and prognostic value in HCC tissue (training cohort, $\mathbf{N}=\mathbf{3 1 8}$ ). Kaplan-Meier curves depiciting OS (a) and TTR (b) for 5-hmC expression, IDH2 expression, and combined 5-hmC/IDH2 expression. I, 5-hmC High/IDH2 High; ll, 5-hmC Low/IDH2 High; III, 5-hmC High/IDH2 Low; IV, 5-hmC Low/IDH2 Low.

recurrence rates in the 5 -hmC ${ }^{\text {Low }} / \mathrm{IDH} 2{ }^{\text {Low }}$ patients were $52.5 \%$ and $71.3 \%$, respectively, which were significantly higher than those in the 5 -hmC ${ }^{\mathrm{High}} / \mathrm{IDH} 2{ }^{\mathrm{High}}$ patients (19.0\% and 36.0\%, respectively) (Figure $3 b)$.

Univariate analysis revealed that 5 -hmC $(P<0.001$ and $P<0.001)$ IDH2 $(P=0.001$ and $P<0.001)$, and 5 -hmC/ IDH2 combined $(P<0.001$ and $P<0.001)$ were associated with OS and TTR. In a multivariate Cox proportional hazards model, 5-hmC and IDH2 were prognostic indicators of OS $(P=0.005$ and $P=0.005)$ and TTR $(P=0.008$ and $P=0.02$ ). When 5 -hmC and IDH2 were combined, we found that 5 -hmC/IDH2 was also an independent prognostic indicator of both OS $(P=0.007)$ and TTR $(P=0.009)$ (Additional file 2: Table S3).

\section{Discussion}

To date, the available data on $5-\mathrm{hmC}$ and IDH2 in HCC have been limited. In this study, we investigated the clinical relevance of 5-hmC and IDH2 protein expression in two large cohorts $(n=646)$ of surgically resected HCCs with 318 cases and 328 cases, respectively.

We determined that high 5-hmC expression was significantly associated with favorable features in HCC patients. This finding may be substantiated by the fact that aggressive histopathological characteristics, including a high AFP level was significantly more frequent in patients with low 5-hmC expression than in those with high expression in training cohort. And a high AFP level, more tumor number, and an advanced TNM staging of $\mathrm{HCC}$ were more detected in those patients with low 5$\mathrm{hmC}$ expression in validation cohort. This indicated that 5 -hmC may be a powerful prognostic indicator in HCC. 5-hmC, an oxidation product of $5 \mathrm{mC}$ via the TET family (which consists of TET1, -2 , and -3 ), is abundant in ES cells and adult neural cells [8]. The relationship between 5 -hmC and tumors is emerging through a number of 
Table 2 Summary of univariate and multivariate analyses of 5-hmC and IDH2 protein expression associated with survival and recurrence in the training cohort $(\mathrm{N}=318)$

\begin{tabular}{|c|c|c|c|c|c|c|c|c|}
\hline \multirow[t]{3}{*}{ Factor } & \multirow{2}{*}{\multicolumn{4}{|c|}{$\begin{array}{c}\text { OS } \\
\text { Multivariate }\end{array}$}} & \multirow{2}{*}{\multicolumn{4}{|c|}{$\begin{array}{c}\text { TTR } \\
\text { Multivariate }\end{array}$}} \\
\hline & & & & & & & & \\
\hline & Univariate $\mathrm{P}$ & Hazard ratio & $95 \% \mathrm{Cl}$ & $\mathrm{P}^{+}$ & Univariate $P$ & Hazard ratio & $95 \% \mathrm{Cl}$ & $\mathrm{P}^{\dagger}$ \\
\hline Sex (female vs. male) & 0.959 & & & NA & 0.083 & & & NA \\
\hline Age, years ( $\leq 50$ vs. $>50$ ) & 0.772 & & & NA & 0.597 & & & NA \\
\hline HBsAg (negative vs. positive) & 0.983 & & & NA & 0.491 & & & NA \\
\hline AFP, ng/ml ( $\leq 20$ vs. $>20)$ & 0.041 & 1.893 & $1.257-2.852$ & 0.002 & 0.230 & & & NA \\
\hline$\gamma$-GT, U/L ( $\leq 54$ vs. $>54)$ & 0.006 & 1.619 & $1.118-2.343$ & 0.011 & 0.003 & 1.547 & $1.138-2.102$ & 0.005 \\
\hline Liver cirrhosis (no vs. yes) & 0.077 & & & NA & 0.009 & 1.824 & $1.135-2.930$ & 0.013 \\
\hline Tumor number (single vs. multiple) & 0.003 & & & NS & 0.002 & 1.651 & $1.135-2.402$ & 0.009 \\
\hline Tumor size, $\mathrm{cm}(\leq 5$ vs. $>5$ ) & 0.009 & & & NS & 0.041 & & & NS \\
\hline Tumor encapsulation (complete vs. none) & 0.261 & & & NA & 0.166 & & & NA \\
\hline Microvascular invasion (no vs. yes) & 0.003 & & & NS & 0.001 & 1.775 & $1.287-2.448$ & $<0.001$ \\
\hline Tumor differentiation (I-II vs. III-IV) & 0.138 & & & NA & 0.053 & & & NA \\
\hline TNM stage (| vs. || III) & $<0.001$ & 2.048 & $1.412-2.971$ & $<0.001$ & $<0.001$ & 1.649 & $1.134-2.397$ & 0.009 \\
\hline 5-hmC (low vs. high) & $<0.001$ & 0.316 & $0.211-0.472$ & $<0.001$ & 0.001 & 0.462 & $0.335-0.636$ & $<0.001$ \\
\hline IDH2 (low vs. high) & $<0.001$ & 0.405 & $0.275-0.594$ & $<0.001$ & 0.006 & 0.591 & $0.432-0.810$ & 0.001 \\
\hline Combination of 5-hmC and IDH2 & $<0.001$ & & & $<0.001$ & $<0.001$ & & & $<0.001$ \\
\hline | versus || & 0.002 & 3.987 & $1.890-8.413$ & $<0.001$ & 0.001 & 2.651 & $1.576-4.461$ & $<0.001$ \\
\hline | versus ||| & 0.002 & 3.359 & $1.607-7.025$ & 0.001 & 0.003 & 2.098 & $1.247-3.530$ & 0.005 \\
\hline I versus IV & $<0.001$ & 8.908 & $4.215-18.825$ & $<0.001$ & $<0.001$ & 3.891 & $2.270-6.671$ & $<0.001$ \\
\hline
\end{tabular}

Abbreviations: OS, overall survival; TTR time to recurrence; AFP, a-fetoprotein; $\mathrm{Y}$-GT, $\mathrm{\gamma}$-glutamyl transferase; TNM, tumor-node-metastasis; Cl, confidence interval; NA, not adopted; NS, not significant.

${ }^{\dagger} \mathrm{Cox}$ proportional hazards regression. Boldface type indicates significant values.

I, 5-hmC High /IDH2 ${ }_{\text {High }}$ Il, 5-hmC Low/IDH2 High; III, 5-hmC High/IDH2 Low; IV, 5-hmC Low/IDH2 Low

studies $[8,11,29]$. In liver cancer research, 5 -hmC expression was decreased in liver cancer compared with the surrounding normal tissue [14,15]. Although previous studies have addressed 5 -hmC protein expression using IHC in archived HCC tissues, the number of cases is limited and lacks further validation. Our study represents the largest analysis of 5 -hmC protein expression in HCC.

We also detected significant correlations between low IDH2 expression and HBsAg background, a high level of AFP, and low-grade tumor differentiation. IDH2, an IDH (which convert isocitrate to $\alpha-K G$ ), is frequently mutated in cancer, particularly in secondary glioblastoma [30], cytogenetically normal acute myeloid leukemia (AML) [31], cartilaginous tumors [32], and intrahepatic cholangiocarcinoma [33]. The pathophysiological function of the $R$-enantiomer of 2-hydroxylglutarate $(R-2-H G)$ is the driving force of IDH1/2 mutation-induced tumorigenesis [22]. In melanoma, IDH2 is frequently downregulated, and the overexpression of IDH2 in a zebrafish melanoma model has been shown to increase the level of $5-\mathrm{hmC}$, resulting in prolonged tumor-free survival [11]. In our group, the preliminary experimental results indicated a tumor suppressor role for IDH2 in HCC (unpublished data); however, the expression of mutated IDH2, the mechanisms of IDH2 mutation, and the precise role of IDH2 in HCC remain under investigation.

One of most notable findings of our study was that the expression of $5-\mathrm{hmC}$ or IDH2 alone, as well as the expression of the combination of $5-\mathrm{hmC}$ and IDH2, was significantly correlated with OS and TTR in two cohorts. Thus, we made a direct comparison of prognosis between four subgroups (5-hmC ${ }^{\mathrm{High}} / \mathrm{IDH} 2{ }^{\mathrm{High}}$, 5-hmC Low/IDH2 High, 5-hmC High/IDH2 Low, and $5-\mathrm{hmC}^{\mathrm{Low}} / \mathrm{IDH} 2^{\mathrm{Low}}$ ) in the training cohort. As expected, patients with $5-\mathrm{hmC}^{\mathrm{High}} / \mathrm{IDH} 2{ }^{\mathrm{High}}$ expression had a significantly better OS and TTR than the patients in the other 3 groups in both univariate and multivariate analyses. These interesting observations were confirmed in a second cohort (validation cohort) that exhibited clinical-pathological features similar to the first cohort (training cohort).

In addition to genetic alterations, epigenetic alterations were also considered to participate in carcinogenesis [34]. It is also plausible that the two mechanisms can coexist and interact, giving birth to the observed hot-spot tumor heterogeneity $[35,36]$. The mechanisms of this interaction are currently the chief investigational pursuit 

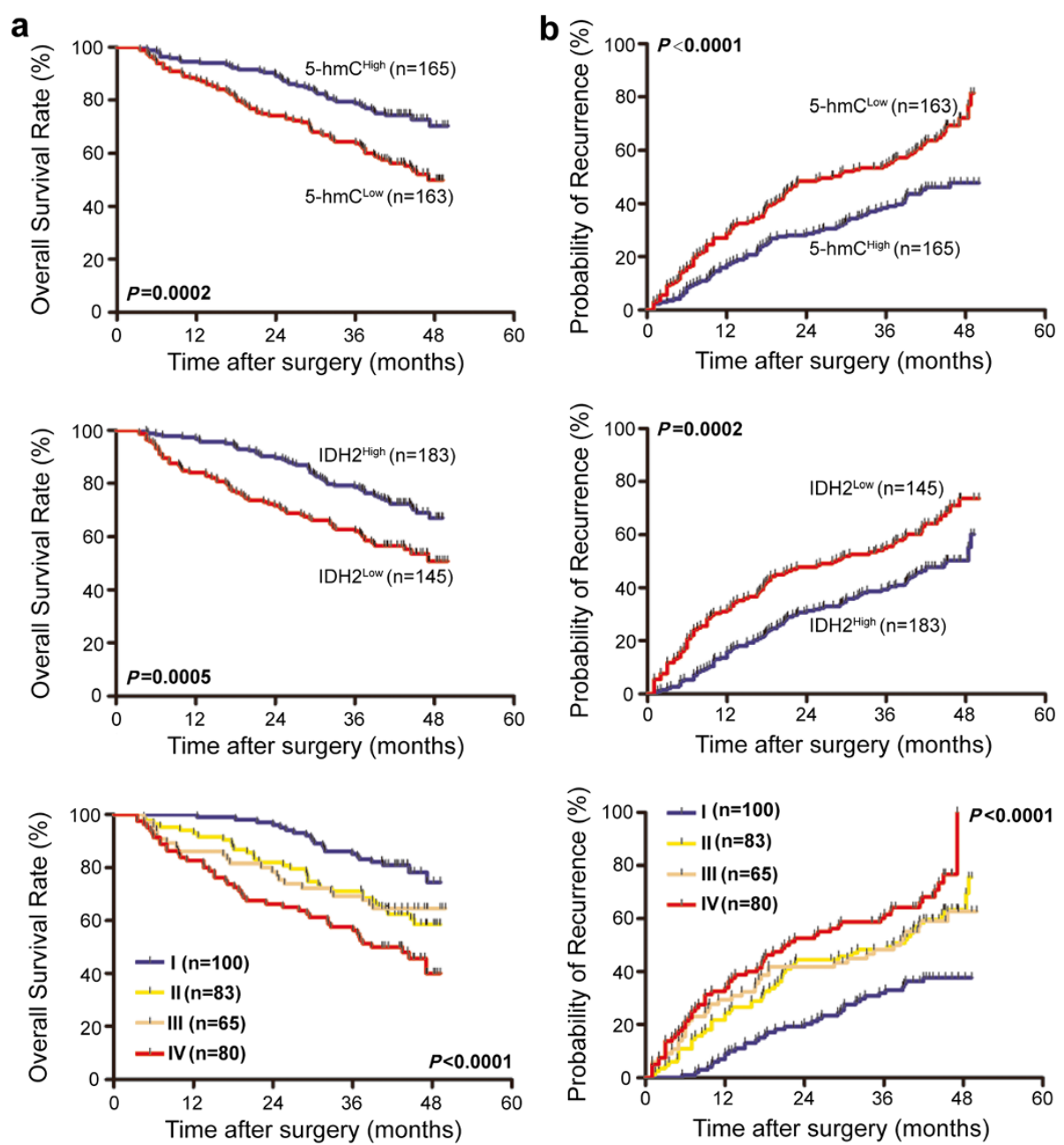

Figure $3 \mathbf{5 - h m C}$ and IDH2 expression and prognostic value in HCC tissue (validation cohort, $\mathbf{N}=\mathbf{3 2 8}$ ). Kaplan-Meier curves depiciting OS

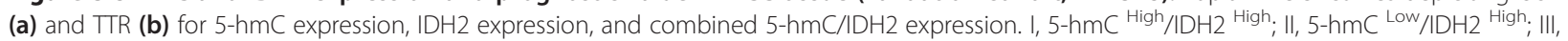
5-hmC ${ }^{\text {High }} / \mathrm{IDH} 2{ }^{\text {Low; }}$ IV, 5-hmC Low/IDH2 Low.

of our laboratory. In this study, we determined the prognostic value of 5-hmC and IDH2 in $\mathrm{HCC}$; further investigations are in progress.

The major limitation of the present work was its retrospective nature. Moreover, it is noteworthy that most HCC patients in China have a hepatitis B virus-positive background, which differs from studies in Japan, Europe, and the United States.

To the best of our knowledge, this is the first paper demonstrating the implications of $5-\mathrm{hmC}$ and IDH2 in HCC. Our findings indicate that a high expression of 5hmC and IDH2 predicts comparably less aggressive tumor behavior. Importantly, 5-hmC expression (particularly when combined with IDH2 expression) enables us to more accurately predict the true prognosis of $\mathrm{HCC}$ patients. Moreover, given the proposed epigenetic nature of 5-hmC and IDH2, the therapeutic manipulation of 5$\mathrm{hmC}$ and IDH2 will assist in guiding clinical strategies.

\section{Conclusions}

In summary, 5-hmC and IDH2 correlate with less aggressive tumor behavior in HCC. Low 5-hmC or IDH2 expression alone and combined 5-hmC and IDH2 expression were associated with lower OS rates and higher cumulative recurrence rates. When 5 -hmC and IDH2 are considered together, they serve as a prognostic marker in patients with surgically resected HCCs.

\section{Additional files}

Additional file 1: Figure S1. Diagram figure to summarize the biological functions of IDH2 and 5-hmC.

Additional file 2: Table S1. Summary of the clinicopathological features of the training and validation cohort. Table S2. Summary of the correlations of 5-hmC and IDH2 protein expression with clinicopathological features in validation cohort $(\mathrm{N}=328)$. Table $\mathbf{S 3}$. Summary of univariate and multivariate analyses of 5-hmC and IDH2 protein expression associated with survival and recurrence in validation cohort $(\mathrm{N}=328)$. 


\section{Abbreviations}

5-hmC: 5-hydroxymethylcytosine; IDH2: Isocitrate dehydrogenase 2; HCC: Hepatocellular carcinoma; OS: Overall survival; TTR: Time to recurrence; TMA: Tissue microarray.

\section{Competing interests}

The authors declare no competing interests.

\section{Authors' contributions}

WRL and MXT contributed equally to this work. All authors read and approved the final manuscript.

\section{Acknowledgments}

Financial support by the grants from National Natural Science Foundation of China (No.81272389, 81030038); National Key Sci-Tech Project

(2012ZX10002011-002); And Scholarship Award for Excellent Doctoral Student granted by Ministry of Education (JFF152005).

\section{Author details}

'Department of Liver Surgery, Liver Cancer Institute, Zhongshan Hospital, Fudan University, 180 FengLin Road, Shanghai 200032, China. ${ }^{2}$ Key Laboratory of Carcinogenesis and Cancer Invasion of Ministry of Education, Zhongshan Hospital, Fudan University, 180 FengLin Road, Shanghai, China. ${ }^{3}$ Institutes of Biomedical Sciences, Fudan University, Shanghai, People's Republic of China.

Received: 7 March 2014 Accepted: 27 March 2014 Published: 10 April 2014

\section{References}

1. El-Serag HB: Hepatocellular carcinoma. N Engl J Med 2011, 365:1118-1127.

2. Maluccio M, Covey A: Recent progress in understanding, diagnosing, and treating hepatocellular carcinoma. CA Cancer J Clin 2012, 62:394-399.

3. Rodriguez-Paredes M, Esteller M: Cancer epigenetics reaches mainstream oncology. Nat Med 2011, 17:330-339.

4. Liu WR, Shi YH, Peng YF, Fan J: Epigenetics of hepatocellular carcinoma: a new horizon. Chin Med J 2012, 125:2349-2360.

5. Berdasco M, Esteller M: Aberrant epigenetic landscape in cancer: how cellular identity goes awry. Dev Cell 2010, 19:698-711.

6. Liu X, Chen X, Yu X, Tao Y, Bode AM, Dong Z, Cao Y: Regulation of microRNAs by epigenetics and their interplay involved in cancer. J Exp Clin Cancer Res 2013, 32:96

7. Tahiliani M, Koh KP, Shen Y, Pastor WA, Bandukwala H, Brudno Y, Agarwal S, lyer LM, Liu DR, Aravind L, Rao A: Conversion of 5-methylcytosine to 5-hydroxymethylcytosine in mammalian DNA by MLL partner TET1. science 2009, 324:930-935.

8. Kriaucionis $\mathrm{S}$, Heintz N: The nuclear DNA base 5-hydroxymethylcytosine is present in Purkinje neurons and the brain. Science 2009, 324:929-930.

9. Ito S, D'Alessio AC, Taranova OV, Hong K, Sowers LC, Zhang Y: Role of Tet proteins in $5 \mathrm{mC}$ to $5 \mathrm{hmC}$ conversion, ES-cell self-renewal and inner cell mass specification. Nature 2010, 466:1129-1133.

10. Szwagierczak A, Bultmann S, Schmidt CS, Spada F, Leonhardt H: Sensitive enzymatic quantification of 5-hydroxymethylcytosine in genomic DNA. Nucleic Acids Res 2010, 38:e181.

11. Lian CG, Xu Y, Ceol C, Wu F, Larson A, Dresser K, Xu W, Tan L, Hu Y, Zhan Q, Lee CW, Hu D, Lian BQ, Kleffel S, Yang Y, Neiswender J, Khorasani AJ, Fang R, Lezcano C, Duncan LM, Scolyer RA, Thompson JF, Kakavand H, Houvras Y, Zon LI, Mihm MC Jr, Kaiser UB, Schatton T, Woda BA, Murphy GF: Loss of 5-hydroxymethylcytosine is an epigenetic hallmark of melanoma. Cell 2012, 150:1135-1146.

12. Orr BA, Haffner MC, Nelson WG, Yegnasubramanian S, Eberhart CG: Decreased 5-hydroxymethylcytosine is associated with neural progenitor phenotype in normal brain and shorter survival in malignant glioma. PloS One 2012, 7:e41036.

13. Kudo Y, Tateishi K, Yamamoto K, Yamamoto S, Asaoka Y, ljichi H, Nagae G, Yoshida $H$, Aburatani $H$, Koike $K$ : Loss of 5-hydroxymethylcytosine is accompanied with malignant cellular transformation. Cancer Sci 2012, 103:670-676.

14. Yang H, Liu Y, Bai F, Zhang JY, Ma SH, Liu J, Xu ZD, Zhu HG, Ling ZQ, Ye D, Guan $\mathrm{KL}$, Xiong $Y$ : Tumor development is associated with decrease of TET gene expression and 5-methylcytosine hydroxylation. Oncogene 2013, 32:663-669.

15. Jin SG, Jiang Y, Qiu R, Rauch TA, Wang Y, Schackert G, Krex D, Lu Q, Pfeifer GP: 5-Hydroxymethylcytosine is strongly depleted in human cancers but its levels do not correlate with IDH1 mutations. Cancer Res 2011, 71:7360-7365.

16. Chen ML, Shen F, Huang W, Qi JH, Wang Y, Feng YQ, Liu SM, Yuan BF: Quantification of 5-methylcytosine and 5-hydroxymethylcytosine in genomic DNA from hepatocellular carcinoma tissues by capillary hydrophilic-interaction liquid chromatography/quadrupole TOF mass spectrometry. Clin Chem 2013, 59:824-832.

17. Reitman ZJ, Jin G, Karoly ED, Spasojevic I, Yang J, Kinzler KW, He Y, Bigner $D D$, Vogelstein $B$, Yan $H$ : Profiling the effects of isocitrate dehydrogenase 1 and 2 mutations on the cellular metabolome. Proc Natl Acad Sci U S A 2011, 108:3270-3275.

18. Wang F, Travins J, DeLaBarre B, Penard-Lacronique V, Schalm S, Hansen E, Straley K, Kernytsky A, Liu W, Gliser C, Yang H, Gross S, Artin E, Saada V, Mylonas E, Quivoron C, Popovici-Muller J, Saunders JO, Salituro FG, Yan S, Murray S, Wei W, Gao Y, Dang L, Dorsch M, Agresta S, Schenkein DP, Biller SA, Su SM, de Botton S: Targeted inhibition of mutant IDH2 in leukemia cells induces cellular differentiation. Science 2013, 340:622-626.

19. Lokody I: Metabolism: IDH2 drives cancer in vivo. Nat Rev Cancer 2013, 13:756-757.

20. Lee D, Kang SY, Suh YL, Jeong JY, Lee Jl, Nam DH: Clinicopathologic and genomic features of gliosarcomas. J Neurooncol 2012, 107:643-650.

21. Wang Z, Bao Z, Yan W, You G, Wang Y, Li X, Zhang W: Isocitrate dehydrogenase 1 (IDH1) mutation-specific microRNA signature predicts favorable prognosis in glioblastoma patients with IDH1 wild type. J Exp Clin Cancer Res 2013, 32:59.

22. Xu W, Yang $H$, Liu $Y$, Yang $Y$, Wang $P$, Kim SH, Ito S, Yang $C$, Wang $P$, Xiao MT, Liu LX, Jiang WQ, Liu J, Zhang JY, Wang B, Frye S, Zhang Y, Xu YH, Lei QY, Guan KL, Zhao SM, Xiong Y: Oncometabolite 2-hydroxyglutarate is a competitive inhibitor of alpha-ketoglutarate-dependent dioxygenases. Cancer Cell 2011, 19:17-30.

23. Gao Q, Qiu SJ, Fan J, Zhou J, Wang XY, Xiao YS, Xu Y, Li YW, Tang ZY: Intratumoral balance of regulatory and cytotoxic $T$ cells is associated with prognosis of hepatocellular carcinoma after resection. J Clin Oncol 2007, 25:2586-2593.

24. Liao R, Sun J, Wu H, Yi Y, Wang JX, He HW, Cai XY, Zhou J, Cheng YF, Fan J, Qiu SJ: High expression of IL-17 and IL-17RE associate with poor prognosis of hepatocellular carcinoma. J Exp Clin Cancer Res 2013, 32:3.

25. Sun HC, Zhang W, Qin LX, Zhang BH, Ye QH, Wang L, Ren N, Zhuang PY, Zhu XD, Fan J, Tang ZY: Positive serum hepatitis $B$ e antigen is associated with higher risk of early recurrence and poorer survival in patients after curative resection of hepatitis B-related hepatocellular carcinoma. $J$ Hepatol 2007, 47:684-690.

26. Shi YH, Ding WX, Zhou J, He JY, Xu Y, Gambotto AA, Rabinowich H, Fan J, Yin XM: Expression of X-linked inhibitor-of-apoptosis protein in hepatocellular carcinoma promotes metastasis and tumor recurrence. Hepatology 2008, 48:497-507.

27. Ding ZB, Shi YH, Zhou J, Qiu SJ, Xu Y, Dai Z, Shi GM, Wang XY, Ke AW, Wu $B$, Fan J: Association of autophagy defect with a malignant phenotype and poor prognosis of hepatocellular carcinoma. Cancer Res 2008, 68:9167-9175

28. Tsukada T, Fushida S, Harada S, Terai S, Yagi Y, Kinoshita J, Oyama K, Tajima $H$, Fujita $H$, Ninomiya I, Fujimura T, Ohta T: Adiponectin receptor-1 expression is associated with good prognosis in gastric cancer. $J$ Exp Clin Cancer Res 2011, 30:107.

29. Li Z, Cai X, Cai CL, Wang J, Zhang W, Petersen BE, Yang FC, Xu M: Deletion of Tet2 in mice leads to dysregulated hematopoietic stem cells and subsequent development of myeloid malignancies. Blood 2011, 118:4509-4518.

30. Zhang C, Moore LM, Li X, Yung WK, Zhang W: IDH1/2 mutations target a key hallmark of cancer by deregulating cellular metabolism in glioma. Neuro Oncol 2013, 15:1114-1126.

31. Wang JH, Chen WL, Li JM, Wu SF, Chen TL, Zhu YM, Zhang WN, Li Y, Qiu $Y P$, Zhao AH, Mi JQ, Jin J, Wang YG, Ma QL, Huang $H$, Wu DP, Wang QR, Li Y, Yan XJ, Yan JS, Li JY, Wang S, Huang XJ, Wang BS, Jia W, Shen Y, Chen Z, Chen SJ: Prognostic significance of 2-hydroxyglutarate levels in acute myeloid leukemia in China. Proc Natl Acad Sci U S A 2013, 110:17017-17022

32. Amary MF, Bacsi K, Maggiani F, Damato S, Halai D, Berisha F, Pollock R, O'Donnell P, Grigoriadis A, Diss T, Eskandarpour M, Presneau N, Hogendoorn 
PC, Futreal A, Tirabosco R, Flanagan AM: IDH1 and IDH2 mutations are frequent events in central chondrosarcoma and central and periosteal chondromas but not in other mesenchymal tumours. J Pathol 2011, 224:334-343.

33. Sia D, Tovar V, Moeini A, Llovet JM: Intrahepatic cholangiocarcinoma: pathogenesis and rationale for molecular therapies. Oncogene 2013, 32:4861-4870.

34. Dawson MA, Kouzarides T: Cancer epigenetics: from mechanism to therapy. Cell 2012, 150:12-27.

35. You JS, Jones PA: Cancer genetics and epigenetics: two sides of the same coin? Cancer Cell 2012, 22:9-20.

36. Meacham CE, Morrison SJ: Tumour heterogeneity and cancer cell plasticity. Nature 2013, 501:328-337.

doi:10.1186/1756-9966-33-32

Cite this article as: Liu et al:: High expression of 5-hydroxymethylcytosine and isocitrate dehydrogenase 2 is associated with favorable prognosis after curative resection of hepatocellular carcinoma. Journal of Experimental \& Clinical Cancer Research 2014 33:32.

\section{Submit your next manuscript to BioMed Central and take full advantage of:}

- Convenient online submission

- Thorough peer review

- No space constraints or color figure charges

- Immediate publication on acceptance

- Inclusion in PubMed, CAS, Scopus and Google Scholar

- Research which is freely available for redistribution 